\title{
Methods Report \\ Nucleofection: A New Method for Cutaneous Gene Transfer?
}

\author{
Frank Jacobsen, Janine Mertens-Rill, Juergen Beller, Tobias Hirsch, Adrien Daigeler, Stefan Langer, \\ Marcus Lehnhardt, Hans-Ulrich Steinau, and Lars Steinstraesser
}

Department of Plastic Surgery, BG University Hospital Bergmannsheil, Ruhr University Bochum, Buerkle-de-la Camp Platz 1, Bochum 44789, Germany

Received 22 May 2006; Revised 3 August 2006; Accepted 24 August 2006

Background. Transfection efficacy after nonviral gene transfer in primary epithelial cells is limited. The aim of this study was to compare transfection efficacy of the recently available method of nucleofection with the established transfection reagent FuGENE6. Methods. Primary human keratinocytes (HKC), primary human fibroblasts (HFB), and a human keratinocyte cell line (HaCaT) were transfected with reporter gene construct by FuGENE6 or Amaxa Nucleofector device. At corresponding time points, $\beta$ galactosidase expression, cell proliferation (MTT-Test), transduction efficiency (X-gal staining), cell morphology, and cytotoxicity (CASY) were determined. Results. Transgene expression after nucleofection was significantly higher in HKC and HFB and detected earlier ( 3 h vs. 24 h) than in FuGENE6. After lipofection $80 \%-90 \%$ of the cells remained proliferative without any influence on cell morphology. In contrast, nucleofection led to a decrease in keratinocyte cell size, with only $20 \%-42 \%$ proliferative cells. Conclusion. Related to the method-dependent increase of cytotoxicity, transgene expression after nucleofection was earlier and higher than after lipofection.

Copyright (c) 2006 Frank Jacobsen et al. This is an open access article distributed under the Creative Commons Attribution License, which permits unrestricted use, distribution, and reproduction in any medium, provided the original work is properly cited.

\section{INTRODUCTION}

The development of gene carriers for efficient gene delivery into cells has attracted growing attention in the recent years. Cutaneous expression can be achieved by genetic manipulation of epidermal keratinocytes ex vivo followed by transplantation or by local delivery of corresponding vectors [1]. Experimental evidence shows that genetically engineered skin can produce and secrete medically relevant proteins to the circulation and also produce enzymes that can clear metabolites accumulating in various diseases [2]. Thus, cutaneous gene transfer approaches may be relevant not only for local skin diseases, but also for certain systemic disorders $[3,4]$.

Virus-mediated delivery of genes is more efficient than plasmid-mediated delivery, but safety concerns regarding immunogenicity, oncogenic properties, risk of recombination with wild-type viruses, and unknown long-term effects remain problematic for its potential clinical use [5]. Another disadvantage of many viral vectors is their limited capacity for delivering large foreign genes. Moreover, viral vectors (eg, $\mathrm{AAV}$, adenovirus, and retrovirus) are difficult to prepare, expensive, and potentially hazardous to work with. Nonviral vectors on the other hand do not have such safety concerns. They remain in a nonreplicative episomal form, have low immunogenicity, and are easy and safe to prepare and use. Furthermore, plasmids may accommodate large fragments of DNA [6].

In contrast to most viral systems, plasmids provide temporary expression of the transfected gene [7]. In order to transfect cells successfully, it is important to overcome the natural barrier of cytoplasmic and nuclear membranes to deliver the genetic material into the cytoplasm or the nucleus. These techniques are categorized into three general groups: direct injection of naked DNA [8], the delivery by a physical method, such as electroporation [9], gene gun [10], microseeding [11], or nucleofection, or mediated by a chemical carrier such as cationic polymers and lipids [12].

The use of these gene delivery methods strictly depended on the kind of target cells, the method of application (eg, if in vivo, ex vivo or in vitro), and the cellular environment.

Compared with other tissues, skin offers the advantage of being easily accessible for manipulations and monitoring. In addition, skin fibroblasts and keratinocytes are interesting candidates for this purpose since they are well-studied primary cells and are relatively easy to isolate and grow in vitro [13], thus permitting self-renovating epithelial transplants by procedures currently used in the treatment of burns or other cutaneous disorders [14]. 
Epidermal keratinocytes are also potentially useful for the production of biologically active factors or polypeptides (eg, growth factors and host defense peptides) for systemic or local delivery to treat inherited or acquired disorders $[15,16]$. For cutaneous gene delivery, techniques must be reproducible, not toxic, and highly efficient to show a biologic effect. The Amaxa nucleofection technique is a variation of electroporation tailored for certain cell types. This electroporation technique has previously shown its transfection efficacy for various primary cells and hard-to-transfect cell lines [17-21].

The goal of this study was to analyze transfection efficacy of reporter genes, cytotoxicity, and cell morphology with nucleofection or cationic transfection reagents in primary human keratinocytes, fibroblasts, and the immortalized keratinocyte cell line HaCaT.

\section{MATERIAL AND METHODS}

\section{Cell culture}

Freshly received human skin was washed in PBS (PAA Laboratories, $\mathrm{H}$ 15002, Linz, Austria) and digested overnight with $0.2 \%$ dispase-solution (4,7 U/mL, Gibco, 17105-041, Paisley, UK). Epidermis was gently peeled off, collected directly into Trypsin/EDTA solution (0.05\%/0.02\%, Gibco, 35400027, Paisley, UK), and incubated at $37^{\circ} \mathrm{C}$ for 5 minutes in a gently shaking water bath. The remaining dermis was used for the isolation of fibroblasts as described below. After trypsin digestion was stopped by adding FBS (HyClone, Logan, USA), the cell suspension was filtered through a 100$\mu \mathrm{m}$ cell strainer (Becton Dickinson Heidelberg, Germany) and centrifuged at $400 \times \mathrm{g}, 4^{\circ} \mathrm{C}$ for 5 minutes. Cells were resuspended in $5 \mathrm{~mL}$ complete keratinocyte medium (containing 3 : 1 DMEM (Gibco, 21969-035, Paisley, UK)), Ham's F12 (Gibco, 21765-029, Paisley, UK), 10\% FBS (Hyclone, Logan, USA), 1\% penicillin/streptomycin (ICN, Aurora, USA), 4 mM L-glutamin (ICN, Aurora, USA), $24.3 \mathrm{mg} / \mathrm{L}$ adenine (Calbiochem, Darmstadt, Germany), $5 \mathrm{mg} / \mathrm{L} \mathrm{In-}$ sulin (Sigma, St Louis, USA), $0.4 \mathrm{mg} / \mathrm{L}$ hydrocortisone (Calbiochem, Darmstadt, Germany), $1.36 \mu \mathrm{g} / \mathrm{L}$ triiodothyronine (Sigma, St Louis, USA), $10^{-10} \mathrm{M}$ cholera toxin (Sigma, St Louis, USA), and $10 \mu \mathrm{g} / \mathrm{L}$ EGF (Sigma, St Louis, USA) and counted by CASY-1 (Schärfe-System, Reutlingen, Germany). Culture flasks precoated with collagen type I were seeded with 75000 cells $/ \mathrm{cm}^{2}$ (Becton Dickinson Falcon, 354236, Heidelberg, Germany). Medium was changed every second day.

\section{Isolation of human fibroblasts}

Excessive dermis from isolation of keratinocytes was washed in PBS (PAA Laboratories, H 15002, Linz, Austria) and weighed in a sterile $50-\mathrm{mL}$ tube. The appropriate amount of sterile filtered collagenase type II (Gibco, Paisley, UK) was added and incubated for 6 hours at $37^{\circ} \mathrm{C}$ in a rotor oven (Bachofer, Reutlingen, Germany). Dissolved der- mis was filtered through a $100 \mu \mathrm{m}$ cell strainer (Becton Dickinson, Heidelberg, Germany) and centrifuged at $400 \times$ g, $4^{\circ} \mathrm{C}$, for 10 minutes. Cells were resuspended in fibroblast medium (containing DMEM (Gibco, 21969-035, Paisley, UK)), 10\% fetal bovine serum (Hyclone, Logan, USA), $1 \%$ penicillin/streptomycin (ICN, Aurora, USA), and $4 \mathrm{mM}$ L-Glutamin (ICN, Aurora, USA) and seeded at a density of 25000 cells $/ \mathrm{cm}^{2}$. Primary human fibroblasts were cultured at $37^{\circ} \mathrm{C}$ in a humidified atmosphere of $5 \% \mathrm{CO}_{2}$, and medium was changed every second day.

\section{Plasmid preparation}

Endotoxin-free Plasmid Purification Kit (Qiagen, Hilden, Germany) was used according to manufacturer's instructions to purify $\mathrm{pCMV} \beta$ gal coding for $\beta$-galactosidase of Escherichia coli and pEGFP-N1 coding for enhanced green fluorescence protein (both Clontech, Heidelberg, Germany). DNA was measured photometrically at a wavelength of $260 \mathrm{~nm}$ and calculated in $\mu \mathrm{g} / \mathrm{mL}$.

\section{Lipofection with FuGENE6}

Cells were seeded at 100000 cells $/ \mathrm{cm}^{2}$ (primary keratinocytes), 52000 cells $/ \mathrm{cm}^{2}$ (primary fibroblasts), and 50000 cells $/ \mathrm{cm}^{2}$ (keratinocyte cell line) in a 6-well plate one day before transfection. The next day, medium was replaced with $1 \mathrm{~mL}$ fresh medium. Three parts FuGENE6 were mixed with two parts plasmid DNA for a final amount of $3 \mu \mathrm{g}$ DNA/well. After 15 minutes of incubation at room temperature, the mixture was added to the cells and further incubated for 6 hours at $37^{\circ} \mathrm{C}, 5 \% \mathrm{CO}_{2}$. Medium was then changed and increased to standard volume.

\section{Nucleofection with Amaxa-Nucleofector}

The same number of cells as described for lipofection was resuspended in corresponding nucleofector solution. DNA and cells were mixed in the Amaxa cuvette and directly placed in the nucleofector device.

Primary human keratinocytes were treated with program T-24, human fibroblasts with U-23, and HaCaT cells with the program U-20. Each program used has been optimized and recommended by the manufacturer. Cell suspension was removed immediately from the cuvette by adding prewarmed medium with the Amaxa pipette. Cell suspension was added to a 6-well plate containing prewarmed medium and incubated at $37^{\circ} \mathrm{C}, 5 \% \mathrm{CO}_{2}$, in a humidified atmosphere.

\section{$\beta$-Galactosidase assay}

Medium was removed and cells were rinsed twice with PBS. Lysis solution was added and a cell scraper was used to detach the cells. Lysed cells were pipetted up and down several times. Lysates were centrifuged at $14000 \mathrm{rpm}, 4^{\circ} \mathrm{C}$, for 2 
minutes (Eppendorf centrifuge 5402, Hamburg, Germany), and the supernatant was snap-frozen in liquid $\mathrm{N}_{2}$ and stored at $-80^{\circ} \mathrm{C}$ until use.

The Galacto-Light-Plus assay (Tropix, Lincoln, USA) was performed according to the manufacturer's instructions. Briefly, $\beta$-galactosidase standards and cell lysates were transferred to a white 96-well plate and incubated with reaction buffer for 30 minutes at room temperature. The injection unit of the microplate luminometer (Berthold, Orion, Pforzheim, Germany) added the accelerator solution automatically, and the signal was measured for 1 second. The final amount of $\beta$-galactosidase in each sample was evaluated by parallel measured $\beta$-galactosidase standard ( $20 \mathrm{ng}-256 \mathrm{fg}$ in a serial dilution).

\section{BCA assay}

To determine the total amount of protein, a BCA protein assay (Pierce, Rockford, USA) was applied according to the manufacturer's protocol, using bovine serum albumin (BSA) as the standard (Pierce, Rockford, USA). In brief, standards and lysates were incubated with working reagent at $37^{\circ} \mathrm{C}$ for 30 minutes. The absorbance was measured at $562 \mathrm{~nm}$ in a microplate ELISA reader (ELX 808, Bio-Tek Instruments, Winooski, USA). The concentrations were determined by a BSA standard curve.

\section{X-gal staining}

Forty-eight hours after transfection, medium was removed, and the cells were washed with PBS. Fixation solution was added for 5 minutes at room temperature, and cells were washed again with $\mathrm{PBS}$. After $\mathrm{X}$-gal staining solution

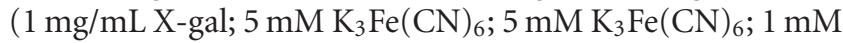
$\mathrm{MgCl}_{2}$; phosphate-buffered saline $\left.\mathrm{pH} 7.4\right)$ was added, cells were incubated for 6 hours at $37^{\circ} \mathrm{C}$. Transfection efficiency was assessed by determining the number of positive cells versus total number of cells in 10 high-power fields (magnification $200 \times$ ).

\section{MTT test}

Twenty-four hours after transfection, MTT (methylthiazolyldiphenyl-tetrazolium bromide, Sigma Aldrich, Taufkirchen, Germany) was added for 1 hour at $37^{\circ} \mathrm{C}$. Medium was removed and cells were lysed by pipetting up and down with $500 \mu \mathrm{L} /$ well $0.01 \mathrm{~N} \mathrm{HCL}+10 \%$ SDS (sodium lauryl sulfate, Sigma Aldrich, Taufkirchen, Germany). The lysates were transferred to a 96-well plate and measured at $562 \mathrm{~nm}$ using an ELISA plate reader (ELX808, BioTek Instruments, Winooski, USA). The number of proliferative cells was correlated with the negative control (100\% survival).

\section{CASY system}

For analysis of cell morphology, both transfection methods were performed with and without DNA and then compared with untreated cells. Cells were washed with PBS and de tached by incubation for 5 minutes at $37^{\circ} \mathrm{C}$ with trypsin/EDTA (0.05\%/0.02\%, PAA Laboratories, Linz, Austria). Cell suspension was diluted 1 : 1000 with CASY Ton (Schärfe System, Reutlingen, Germany) in CASY cups and measured by CASY device. This system counts the amount of cells per volume and calculates the median dimension of the cells by measuring the resistance of the used cell suspension.

\section{Statistical analysis}

All assays were performed in triplicate. Results were calculated in Excel and were statistically evaluated with the software program SPSS 11.0 (SPSS Inc, Chicago, USA). Results were regarded as significant with $P<.05$.

\section{RESULTS}

\section{Transgene expression}

To analyze the transfection efficacy, $\beta$-galactosidase expression was determined in correlation to the total protein. Nucleofection induces high transgene expression rates in primary human keratinocytes (HKC) and fibroblasts (HFB). In primary human fibroblasts gene expression could be measured 12 hours after lipofection, whereas transgene expression was detectable after 3 hours and peaked after 72 hours if nucleofection was used. Lipofection also produced a maximum value at 72 hours $(39 \mathrm{ng} / \mathrm{mg}$ total protein) that was 15 times lower than the expression values of nucleofected cells (600 ng/mg total protein) (Figure 1). Transgene expression decreased drastically to background level for nucleofected fibroblasts between days 3 and 5. During the whole time course, lipofection demonstrated an even progression.

The progression curve of transgene expression demonstrated lower acclivity for human primary keratinocytes than for primary fibroblasts. The measured $\beta$-galactosidase peaked after 72 hours at a level 7 times higher with nucleofection than with FuGENE6 (198 ng/mg vs 27 ng/mg). Transgene expression also proceeded moderately and did not reach background level until day 5 .

In the human keratinocyte cell line HaCaT, only a low expression rate was achieved with both techniques. Lipofection gene expression was detectable after 12 hours. In contrast to the lipofection, which reached peak value after 24 hours (max $7 \mathrm{ng} / \mathrm{mg}$ total protein), the expression after nucleofection increased continuously until 72 hours $(\max 10 \mathrm{ng} / \mathrm{mg}$ total protein). Expression level was significantly $(P<.05)$ higher for lipofection between days 1 and 2, whereas nucleofection achieved significantly $(P<.05)$ higher transgene expression at days 3 and 5 (Figure 1).

\section{Transfection efficiency}

The number of $\beta$-galactosidase-producing cells was determined with $\mathrm{X}$-gal staining and high-power field (HPF) calculation. The calculated transgene-expressing cells were 


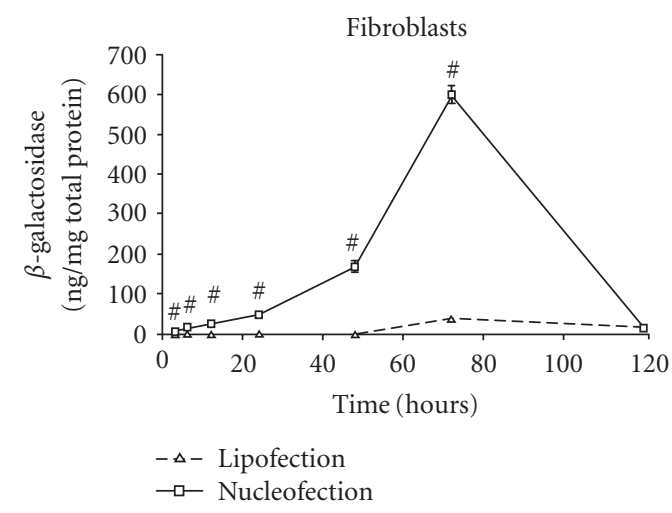

(a)

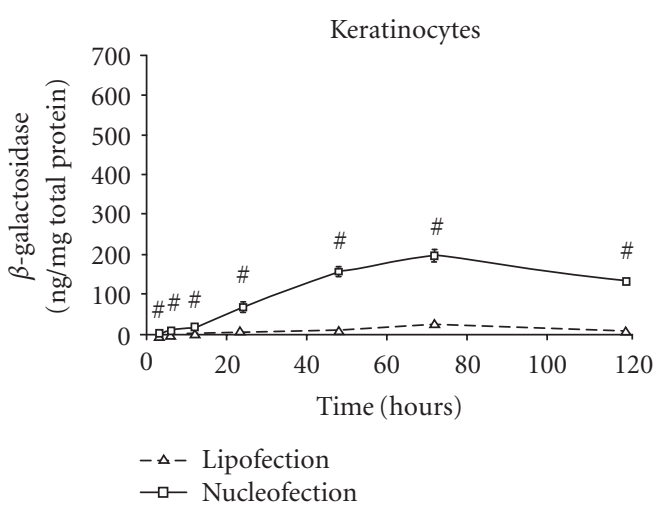

(b)

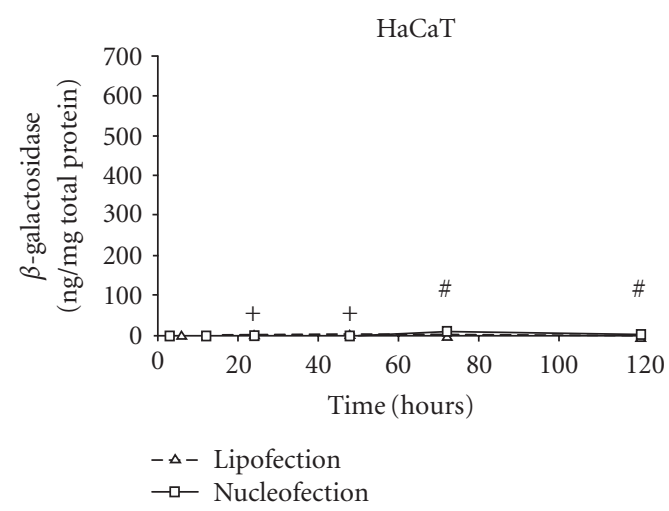

(c)

FIGURE 1: Transfection efficacy. Reporter gene expression in epithelial cells (5 days followup). FuGENE6-enhanced lipofection (open triangle) and Amaxa nucleofection techniques (open square) were compared. Each measured value including the HaCaT curve was upon background levels. \#: $(P<.05)$ nucleofection versus lipofection, +: $(P<.05)$ lipofection versus nucleofection. Data are displayed as mean \pm SEM.

related to the total number of used cells. A transfection rate of $5 \%$ could be demonstrated for human keratinocytes and $10 \%$ for human fibroblasts, whereas the human keratinocyte cell line achieved only $0.5 \%$. For all cases nucleofection demonstrated significantly $(P<.05)$

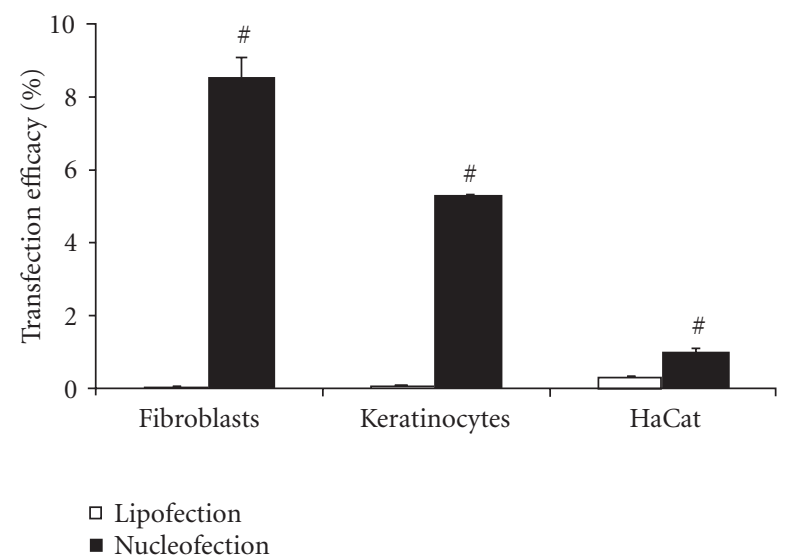

FIgure 2: Histological analysis. The transfection efficacy of the FuGENE6-enhanced lipofection (white bars) and the Amaxa nucleofection techniques (black bars) are displayed. X-gal stained cells were counted in 10 high-power fields (HPF) at 200-fold magnification and extrapolated on positive cells/well. Positive cells were correlated to the number of cells used per well. \#: $(P<.05)$ nucleofection versus lipofection. Data are displayed as mean \pm SEM.

higher transfection efficiency than the FuGENE6 lipofection method (Figure 2). Differences in cellular morphology were not seen after X-gal staining (Figure 3). Cell survival was lower for nucleofection than for lipofection. However, only small cell islands were observed 48 hours after nucleofection, whereas a confluent monolayer was established at the same time-point after lipofection.

\section{Cell viability}

Comparing the effect of both transfection methods on cell viability, the activity of mitochondrial dehydrogenase was determined by MTT test. The cell toxicity induced by the corresponding application was measured for the method alone or together with DNA. This assay revealed high cell viability after FuGENE6 lipofection. The results demonstrated a significant difference in applying $\mathrm{Fu}-$ GENE6 transfection only for HaCaT cells. After lipofection, $75 \%$ of $\mathrm{HaCaT}$ cells, $85 \%$ of keratinocytes, and $99 \%$ of fibroblasts were still proliferating. Nucleofection, however, revealed a proliferation rate $22 \%$ for $\mathrm{HaCaT}$ cells, $23 \%$ for keratinocytes, and $37 \%$ for fibroblasts. Within all experimental groups lipofection resulted in a significantly $(P<.05)$ higher proliferation rate than nucleofection. In addition, HaCaT cells demonstrated a significant $(P<.05)$ decrease of proliferation if DNA was applied (Figure 4).

The cells were analyzed by CASY system to determine whether cell morphology was dependent on the transfection method applied with or without DNA. This system permits determination of cell count and diameter and vol- 


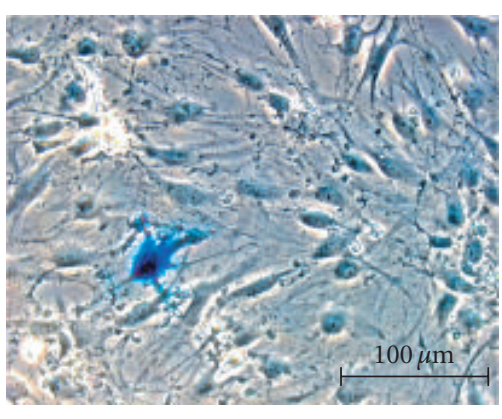

(a)

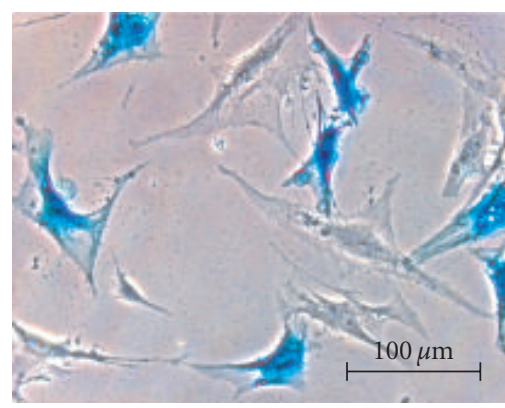

(d)

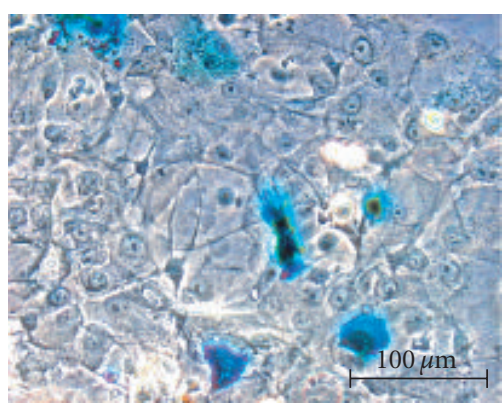

(b)

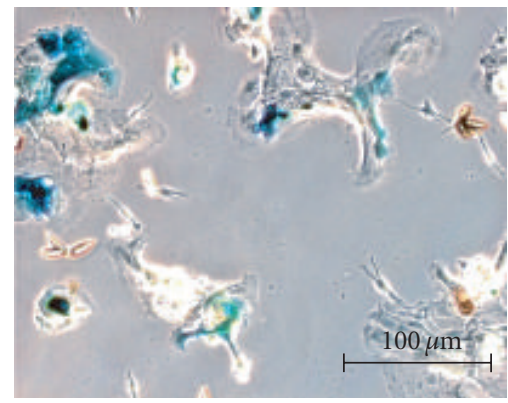

(e)

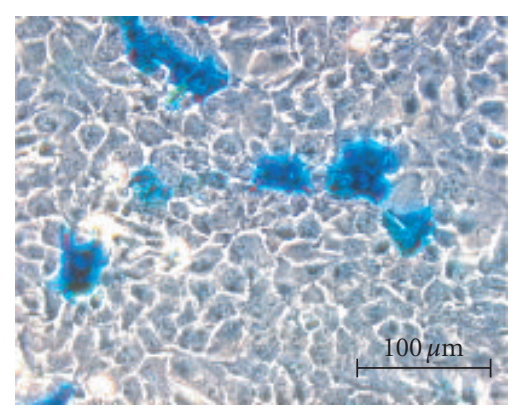

(c)

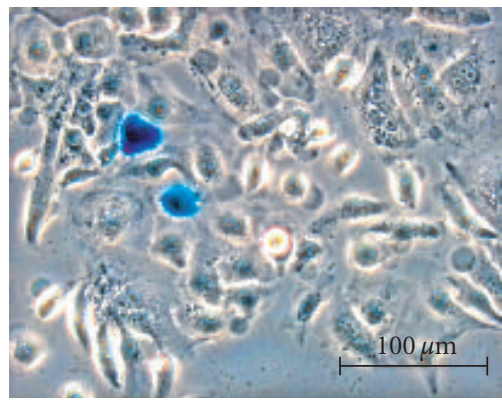

(f)

FIGURe 3: Cellular morphology. X-gal stained primary fibroblasts (a) and (d), primary keratinocytes (b) and (e), and HaCaT cells (c) and (f). Lipofection (a)-(c) and nucleofection techniques (d)-(f) were compared. Pictures were selected to demonstrate typical positively stained cells for both techniques. Pictures shown are not representative in comparison with the corresponding HPF counting of Figure 2 (Bar represents $100 \mu \mathrm{m}$, magnification $200 \times)$.

ume of the cells. Lipofection revealed moderate differences between treated and untreated cells. In general, the resulting curves were similar in appearance, with no stable change in cell diameters and cell counts. On the other hand, nucleofection showed an adjustment of the distribution curve to lower counts for all tested cell types. Further differences were observed in keratinocytes, if DNA was applied, compared with the nucleofection technique alone. With DNA, nucleofection produced smaller cell diameters (Figure 5).

\section{DISCUSSION}

Transient cutaneous gene therapy might be an important treatment option in the near future for wound repair and regeneration $[10,22,23]$, but reliable methods are needed to achieve a level of transgene expression that shows a reproducible biologic effect. In this report, applicability of nucleofection technology for the transient transfection of fibroblasts and keratinocytes is demonstrated. We are considering the application of ex vivo-transfected primary cells as an attractive method for wound healing
[14]. For conventional transfection methods such as calcium phosphate-mediated transfection, lipofection, or electroporation, exponentially growing cells were recommended $[17,24]$, in contrast to nucleofection, in which the proliferation status of the cells has very little impact on the transfection efficacy.

In fact, the transfection efficacy with a maximum of $9 \%$ for primary fibroblasts seems to be low. According to the calculation that determined $1 \%-9 \%$ of positive cells, compared with the number of cells used for each experiment and according to the level of about $20 \%-40 \%$ surviving cells, the transfection efficacy correlated to $5 \%-25 \%$. Higher percentages of transgene-positive keratinocytes have been reported after nucleofection, but in these cases cells were transfected with eGFP-encoding plasmids and results were determined by FACS analysis, which is indisputably the more sensitive technique [25], whereas quantification with bioluminescence is much more sensitive for $\beta$-galactosidase. However, in comparison with viral gene delivery techniques, the transfection rate with nucleofection was moderate, but on a high level if nonviral techniques were taken into consideration [26]. 
The discrepancy between nucleofection (3 hours) and lipofection (24 hours) at the onset of transgene expression may depend on the additional barrier of the nuclear membrane that lipofection has to circumvent before transgene expression starts. The differences in the level of expression after nucleofection and lipofection likely arise from the fact that gene expression is a time-dependent process. Indeed, as soon as the plasmid reached the nucleus directly, $\beta$-galactosidase level increased immediately. Furthermore, since $\beta$-galactosidase is a stable protein with a half-life greater than 24 hours in cells [27], the levels of $\beta$-galactosidase should remain higher in nucleofected cells than in lipofected cells at all times because the DNA is available to the transcription machinery for a longer time. A strong increase of transgene expression was observed in fibroblasts before day 3 , followed by a drastic decrease between days 3 and 5 . This increase may be caused by promoter silencing, intracellular transcript inhibition, or plasmid degradation.

After transfection, proliferation of primary keratinocytes was stronger than that of $\mathrm{HaCaT}$ cells, which may be related to the diversification of primary cell population; nevertheless, the involved mechanism is still unclear. None to moderate inhibition of proliferation was shown for FuGENE6enhanced transfection. The survival rate of primary human skin fibroblasts treated with $3 \mu \mathrm{L}$ FuGENE6 has been reported in the literature at $86 \%$ [28]. Transfection leads to cycle arrest of metabolically active transfected cells (those expressing the transgene), which is not caused by transgene expression per se, but is due to an indirect effect of the exogenous DNA [29]. It has also been shown that higher amounts of foreign DNA lead to a decrease of proliferating and colonizing keratinocytes [24]. The data from our study showed a remarkable variation between primary keratinocytes and the $\mathrm{HaCaT}$ cell line. HaCaT cells are often used as a standard in vitro model to investigate cutaneous processes, because of their availability, genetic homogeneity, and comparable behavior to primary keratinocytes [30-32]. However, we have strong evidence that primary keratinocytes and the $\mathrm{HaCaT}$ cell line are not biologically comparable. There was an obvious difference in cellular morphology and proliferation when lipofection or nucleofection was used. This finding will be of relevance if nucleofection is used as a standardized ex vivo transfection system. The obvious differences in cellular morphology shown by the pictures of Xgal staining are due to methodical differences. For the nucleofection method, detached cells were transfected, wheras attached cells were transfected with the lipofection method. No further differences could be detected between the techniques, but the CASY system showed a shift to lower cell diameters after nucleofection of primary keratinocytes. Cell fusion resulting in polynucleated cells was not observed anywhere. Since the nucleofection technology facilitates transfer of DNA directly into the cell's nucleus, it permits transfection of a wide variety of primary cells and cell lines, which proliferate slowly or are amitotic. Thus, this technology allows the selection of a cell type for gene transfer according to its biological significance rather than to its division rate. It allows experiments of higher biological relevance in many fields of

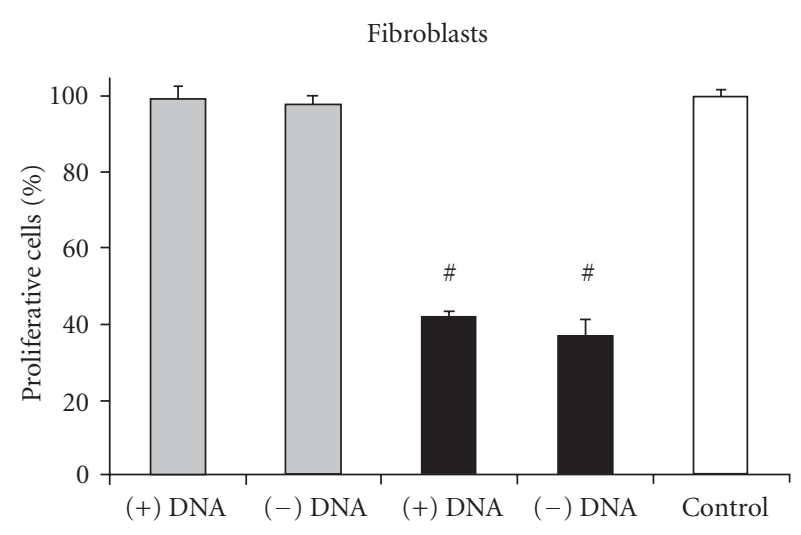

(a)

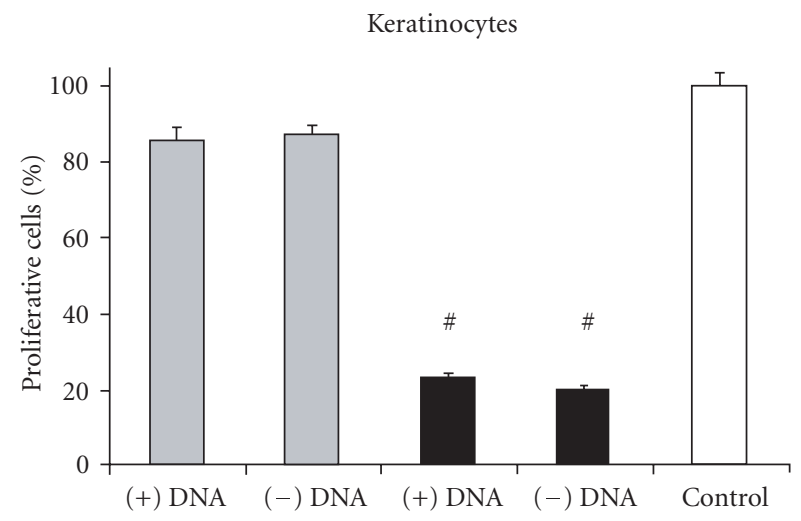

(b)

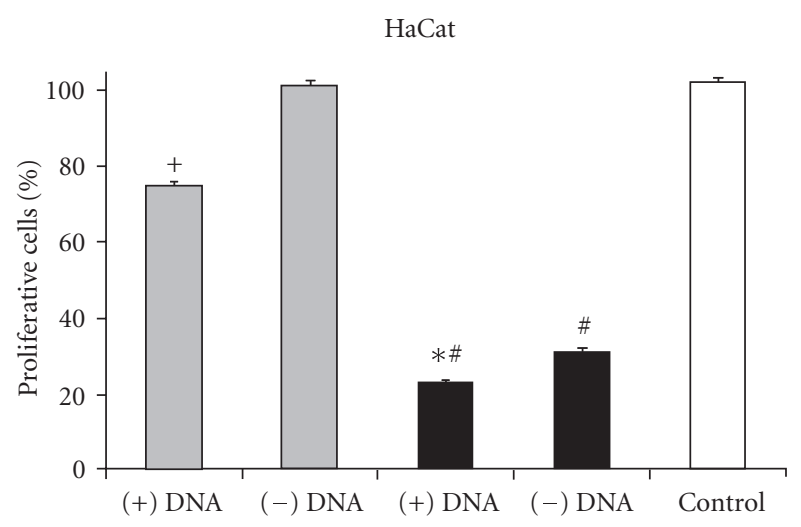

(c)

FIGURe 4: Proliferation. MTT test was performed to analyze the cytotoxic side effects of lipofection (striped) and nucleofection techniques (black). The additional cytotoxic effect of plasmid DNA was determined to distinguish these techniques further. Proliferation control was set at $100 \%$. \#: $(P<.05)$ nucleofection versus lipofection; $*$ : $(P<.05)$ nucleofection $(+)$ DNA versus nucleofection $(-)$ DNA; +: $(P<.05)$ lipofection $(+)$ DNA versus lipofection $(-)$ DNA. Data are shown as mean \pm SEM.

basic research and may be of value in gene-transfer-based industrial approaches, such as functional genomics, gene therapy research, and the development of ex vivo protocols, especially if transient expression is desired. 


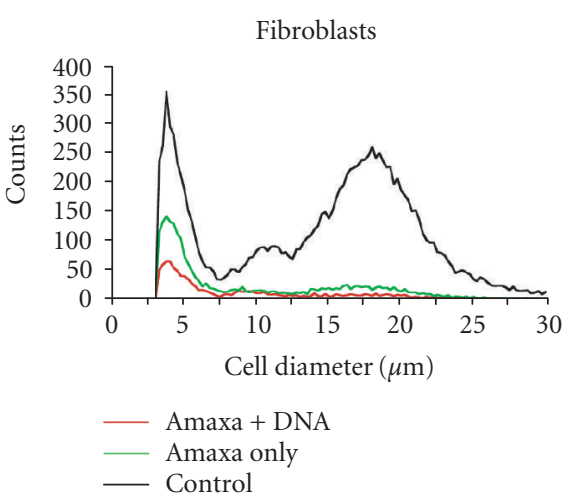

(a)

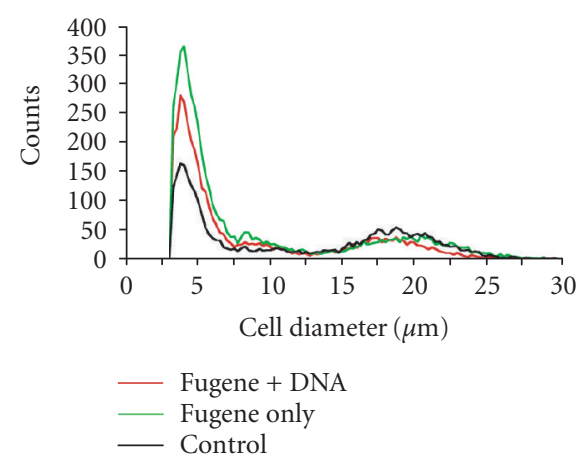

(d)

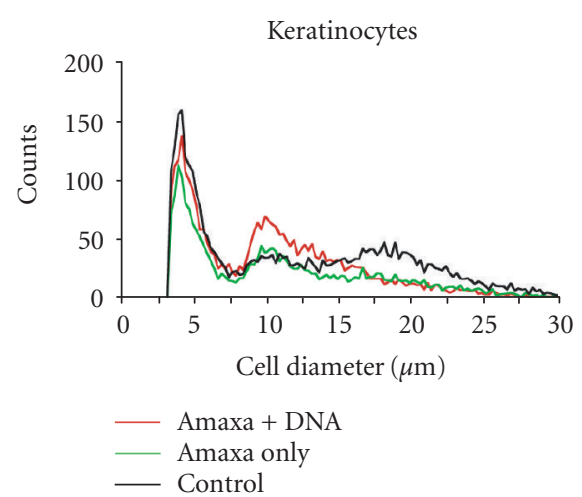

(b)

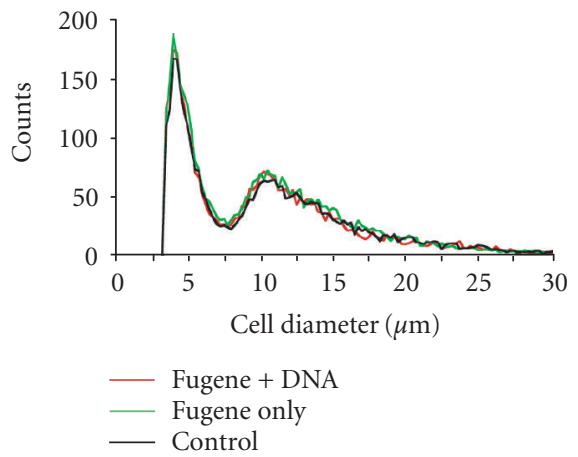

(e)

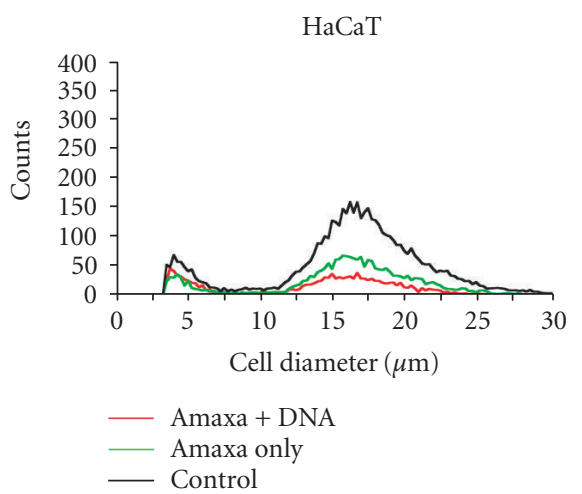

(c)

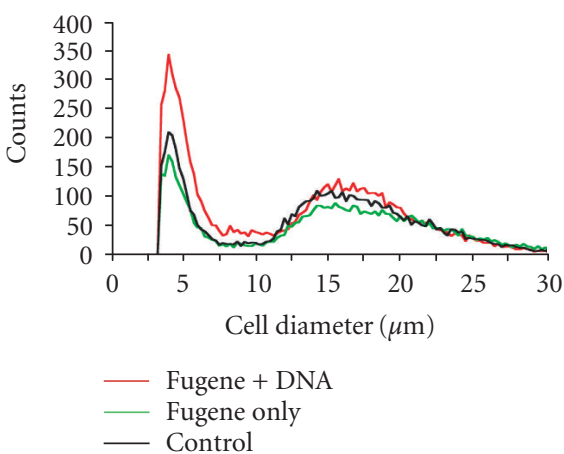

(f)

FIGURe 5: CASY Analysis. Nucleofection (upper panels) and lipofection (lower panels) were correlated by CASY system. Each cell type was treated with (red) or without (green) DNA. Untreated cells served as control (black). Cell counts ( $y$ axis) were plotted against the correlated diameter $(\mu \mathrm{m}, x$ axis). The first peak $(5-7 \mu \mathrm{m})$ represents the amount of cell debris. Further peaks of higher cell diameter within the same plot refer to different levels of cell differentiation. A difference of cell diameter was measured only for nucleofected primary keratinocytes. Data are shown as mean SEM.

\section{ACKNOWLEDGMENTS}

We thank Amaxa for supplying us with the nucleofector, the nucleofector solutions, and technical advice. The work was financially supported in part by the Deutsche Forschungsgemeinschaft (DFG: Ste 1099) and FoRUM (Ruhr-Universität Bochum).

\section{REFERENCES}

[1] Spirito F, Meneguzzi G, Danos O, Mezzina M. Cutaneous gene transfer and therapy: the present and the future. Journal of Gene Medicine. 2001;3(1):21-31.

[2] Cao T, Wang X-J, Roop DR. Regulated cutaneous gene delivery: the skin as a bioreactor. Human Gene Therapy. 2000;11(16):2297-2300.

[3] Khavari PA. Therapeutic gene delivery to the skin. Molecular Medicine Today. 1997;3(12):533-538.

[4] Khavari PA. Genetic correction of inherited epidermal disorders. Human Gene Therapy. 2000;11(16):2277-2282.

[5] Mah C, Byrne BJ, Flotte TR. Virus-based gene delivery systems. Clinical Pharmacokinetics. 2002;41(12):901-911.

[6] Hengge UR, Bardenheuer W. Gene therapy and the skin. American Journal of Medical Genetics Part C: Seminars in Medical Genetics. 2004;131 C(1):93-100.
[7] Niidome T, Huang L. Gene therapy progress and prospects: nonviral vectors. Gene Therapy. 2002;9(24):1647-1652.

[8] Wolff JA, Budker V. The mechanism of naked DNA uptake and expression. Advances in Genetics. 2005;54:3-20.

[9] Rols M-P, Delteil C, Golzio M, Dumond P, Cros S, Teissie J. In vivo electrically mediated protein and gene transfer in murine melanoma. Nature Biotechnology. 1998;16(2):168-171.

[10] Steinstraesser L, Föhn M, Klein RD, et al. Feasibility of biolistic gene therapy in burns. Shock. 2001;15(4):272-277.

[11] Eriksson E, Yao F, Svensjö T, et al. In vivo gene transfer to skin and wound by microseeding. Journal of Surgical Research. 1998;78(2):85-91.

[12] Jacobsen LB, Calvin SA, Colvin KE, Wright M. FuGENE 6 transfection reagent: the gentle power. Methods. 2004;33(2): 104-112.

[13] Normand J, Karasek MA. A method for the isolation and serial propagation of keratinocytes, endothelial cells, and fibroblasts from a single punch biopsy of human skin. In Vitro Cellular and Developmental Biology-Animal. 1995;31(6):447-455.

[14] Chester DL, Balderson DS, Papini RPG. A review of keratinocyte delivery to the wound bed. Journal of Burn Care and Rehabilitation. 2004;25(3):266-275.

[15] Cao T, Tsai SY, O’Malley BW, Wang X-J, Roop DR. The epidermis as a bioreactor: topically regulated cutaneous delivery into the circulation. Human Gene Therapy. 2002;13(9):1075-1080. 
[16] Kopp J, Wang GY, Kulmburg P, et al. Accelerated wound healing by in vivo application of keratinocytes overexpressing KGF. Molecular Therapy. 2004;10(1):86-96.

[17] Gresch O, Engel FB, Nesic D, et al. New non-viral method for gene transfer into primary cells. Methods. 2004;33(2):151-163.

[18] Hamm A, Krott N, Breibach I, Blindt R, Bosserhoff AK. Efficient transfection method for primary cells. Tissue Engineering. 2002;8(2):235-245.

[19] Lenz P, Bacot SM, Frazier-Jessen MR, Feldman GM. Nucleoporation of dendritic cells: efficient gene transfer by electroporation into human monocyte-derived dendritic cells. FEBS Letters. 2003;538(1-3):149-154.

[20] Martinet W, Schrijvers DM, Kockx MM. Nucleofection as an efficient nonviral transfection method for human monocytic cells. Biotechnology Letters. 2003;25(13):1025-1029.

[21] Trompeter H-I, Weinhold S, Thiel C, Wernet P, Uhrberg M. Rapid and highly efficient gene transfer into natural killer cells by nucleofection. Journal of Immunological Methods. 2003;274(1-2):245-256.

[22] Jacobsen F, Mittler D, Hirsch T, et al. Transient cutaneous adenoviral gene therapy with human host defense peptide hCAP18/LL-37 is effective for the treatment of burn wound infections. Gene Therapy. 2005;12(20):1494-1502.

[23] Khavari PA, Rollman O, Vahlquist A. Cutaneous gene transfer for skin and systemic diseases. Journal of Internal Medicine. 2002;252(1):1-10.

[24] Batard P, Jordan M, Wurm F. Transfer of high copy number plasmid into mammalian cells by calcium phosphate transfection. Gene. 2001;270(1-2):61-68.

[25] Chen M, Li W, Fan J, Kasahara N, Woodley D. An efficient gene transduction system for studying gene function in primary human dermal fibroblasts and epidermal keratinocytes. Clinical and Experimental Dermatology. 2003;28(2):193-199.

[26] Jacobsen F, Hirsch T, Mittler D, et al. Polybrene improves transfection efficacy of recombinant replication-deficient adenovirus in cutaneous cells and burned skin. Journal of Gene Medicine. 2006;8(2):138-146.

[27] Smith RL, Geller AI, Escudero KW, Wilcox CL. Long-term expression in sensory neurons in tissue culture from herpes simplex virus type 1 (HSV-1) promoters in an HSV-1-derived vector. Journal of Virology. 1995;69(8):4593-4599.

[28] Hellgren I, Drvota V, Pieper R, et al. Highly efficient cell-mediated gene transfer using-non-viral vectors and FuGene ${ }^{\mathrm{TM}} 6$ : in vitro and in vivo studies. Cellular and Molecular Life Sciences. 2000;57(8-9):1326-1333.

[29] Jensen UB, Petersen MS, Lund TB, Jensen TG, Bolund L. Transgene expression in human epidermal keratinocytes: cell cycle arrest of productively transfected cells. Experimental Dermatology. 2000;9(4):298-310.

[30] Compton SH, Mecklenbeck S, Mejia JE, et al. Stable integration of large (> $100 \mathrm{~kb}) \mathrm{PAC}$ constructs in HaCaT keratinocytes using an integrin-targeting peptide delivery system. Gene Therapy. 2000;7(18):1600-1605.

[31] Gault N, Vozenin-Brotons MC, Calenda A, Lefaix JL, Martin MT. Promoter sequences involved in transforming growth factor $\beta 1$ gene induction in HaCat keratinocytes after gamma irradiation. Radiation Research. 2002;157(3):249-255.

[32] Sumiyoshi K, Nakao A, Setoguchi Y, Tsuboi R, Okumura K, Ogawa H. TGF- $\beta /$ Smad signaling inhibits IFN- $\gamma$ and TNF- $\alpha$ induced TARC (CCL17) production in HaCaT cells. Journal of Dermatological Science. 2003;31(1):53-58. 

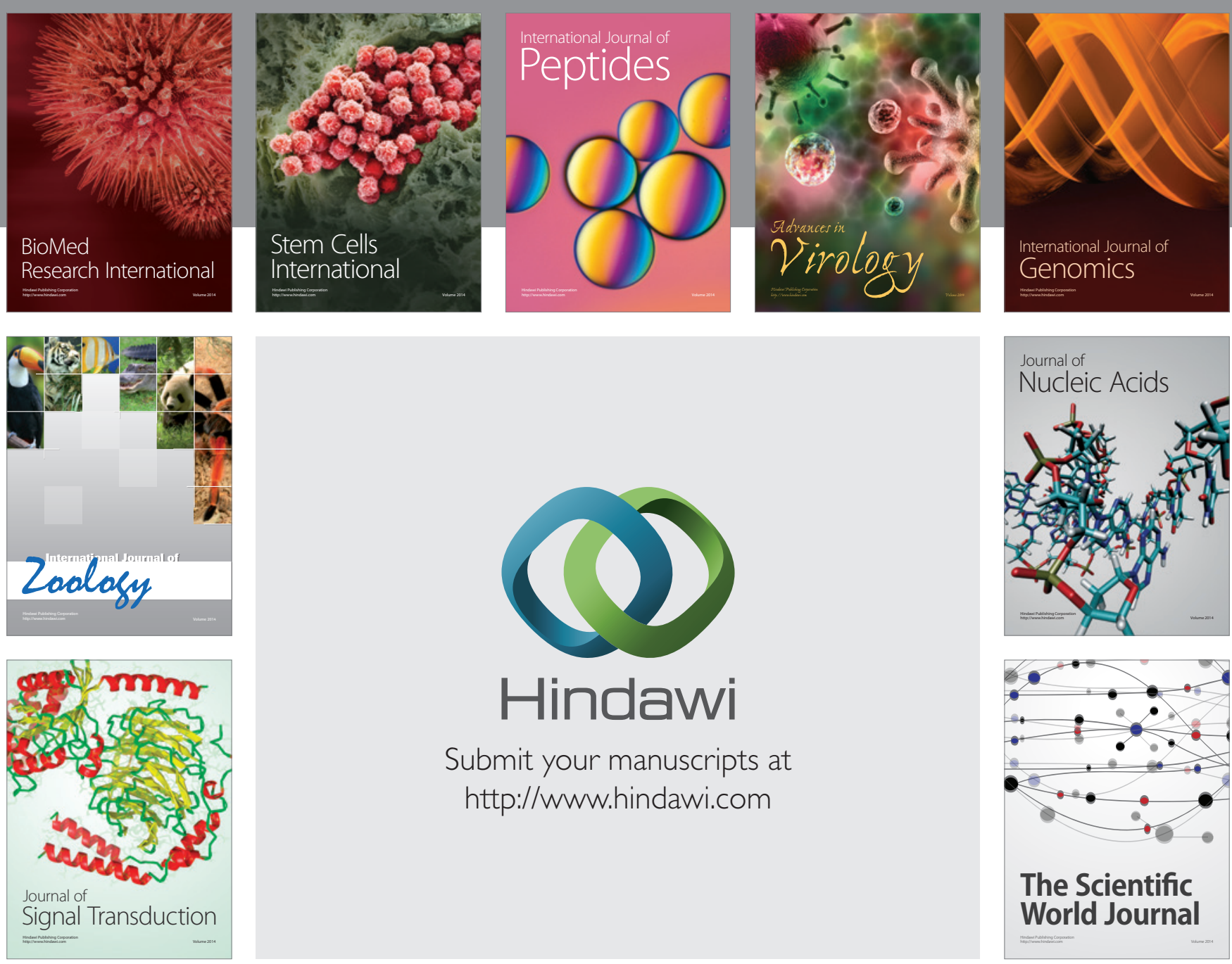

Submit your manuscripts at

http://www.hindawi.com
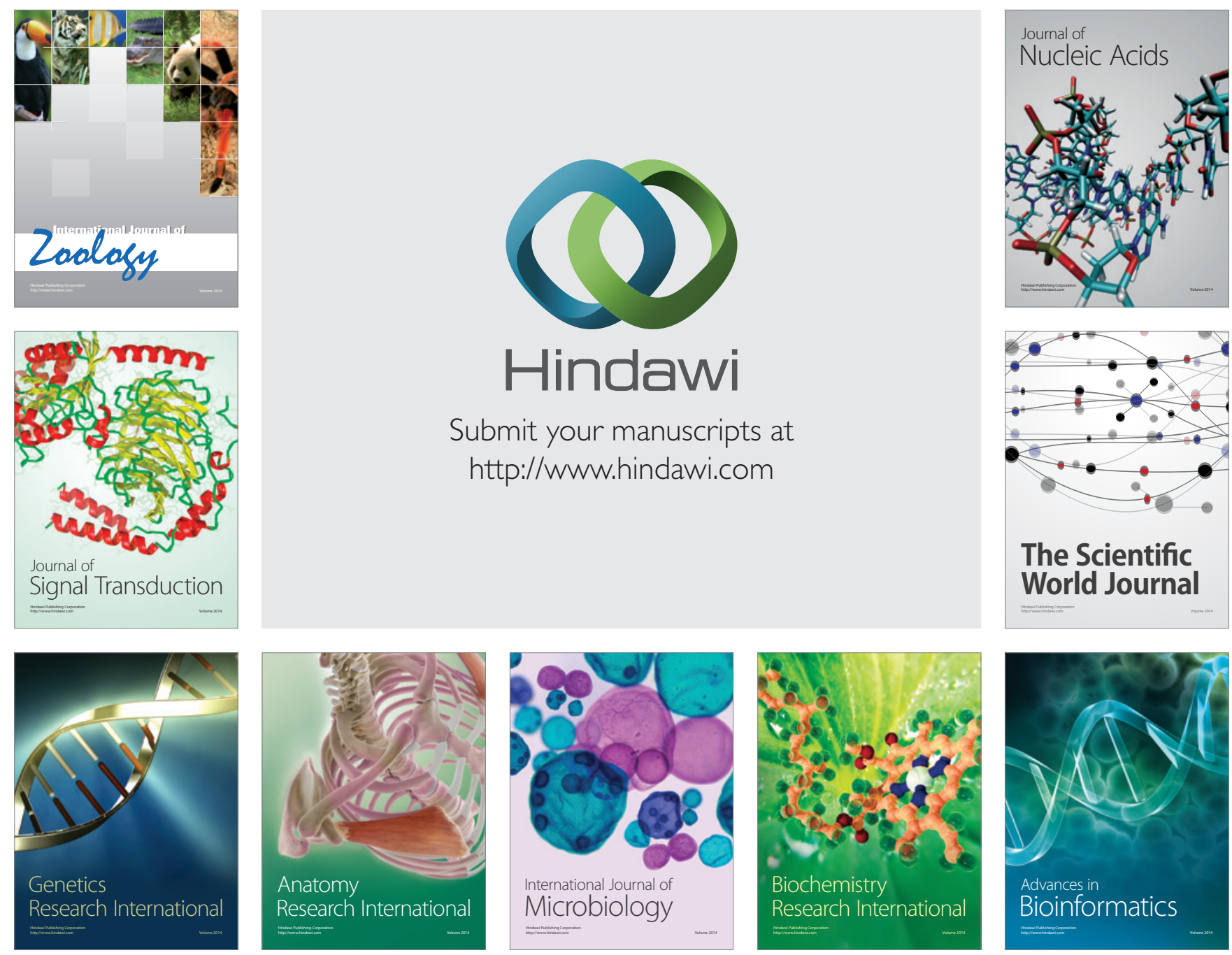

The Scientific World Journal
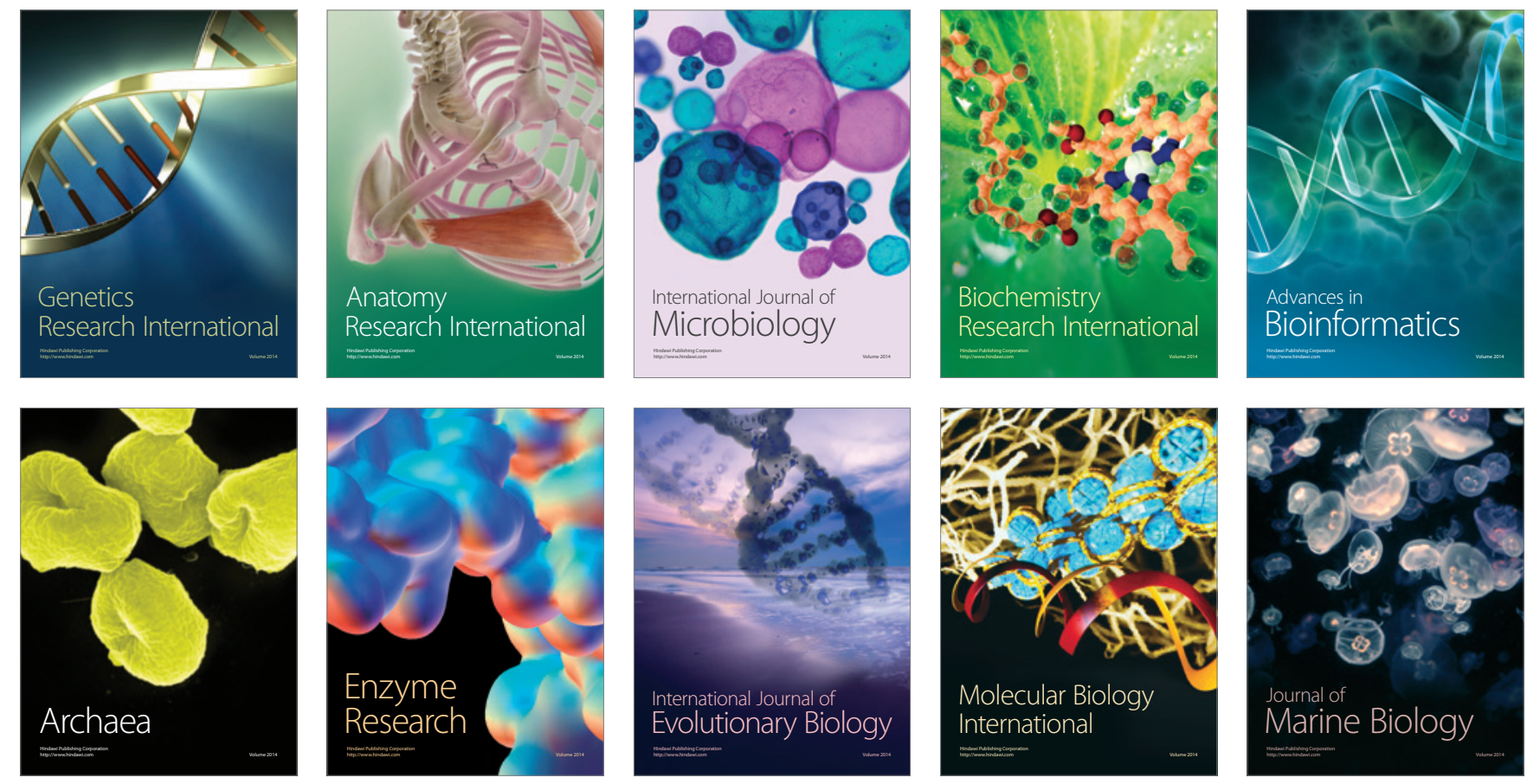